\title{
Position and Thickness Optimization of Ribs for Ventilation Covering Using the Micro Genetic Algorithm with an Interpolated Smooth Objective Function
}

\author{
Young-Doo Kwon, ${ }^{1}$ Soon-Bum Kwon, ${ }^{1}$ Seong-Su Kim, ${ }^{2}$ and Jin-Won Lee ${ }^{2}$ \\ ${ }^{1}$ Department of Mechanical Engineering \& IEDT, Kyungpook National University, Daegu 702-701, Republic of Korea \\ ${ }^{2}$ Department of Mechanical Engineering, Graduate School of Kyungpook National University, Daegu 702-701, Republic of Korea \\ Correspondence should be addressed to Young-Doo Kwon; ydkwon@knu.ac.kr
}

Received 27 February 2015; Revised 17 July 2015; Accepted 26 July 2015

Academic Editor: Alessandro Gasparetto

Copyright ( $\odot 2015$ Young-Doo Kwon et al. This is an open access article distributed under the Creative Commons Attribution License, which permits unrestricted use, distribution, and reproduction in any medium, provided the original work is properly cited.

\begin{abstract}
Structures with supporting ribs are adopted in many fields of engineering. These ribs are attached to the main plate or shell to increase stiffness and reduce the stresses of a structure. Currently, much research in structural optimization has been devoted to size or thickness optimizations. In this study, the discrete positions of the ribs of a structure are optimized in addition to their thicknesses. The objective function, which is the total weight of a structure, is a continuous function with respect to the thickness of the ribs. However, it is a stepwise function of a dimensionless variable, which represents the set of positions of the ribs. Because of this stepwise objective function, the gradient method of optimization is not applicable. Therefore, we applied the micro genetic algorithm (MGA), which does not need derivatives of the objective function. To accelerate the rate of convergence, the stepwise objective function is interpolated to a smooth artificial objective function that does not alter the optimal solution.
\end{abstract}

\section{Introduction}

With the recent construction of large-scale buildings, there has been an increase in the number of ventilation facilities for the ventilation of underground spaces with a dramatic increase in the underground sector. The entrances for ventilation shafts are installed above ground, and most of these ventilation shafts are located on sidewalks or in parks, which are easily accessible to pedestrians. Moreover, the heights of most shafts are easily accessible to passersby. Ventilation shafts consist of steel coverings and ribs. As studied by Lee et al. [1], steel coverings are structures that mainly function as drainage covers, as they enhance the strength of the structure and facilitate efficient drainage through the formation of a tilted inflow tract for wastewater or rainfall. Furthermore, these covers enable smooth discharge and inflow of air through large openings of ventilation holes, which prevents a loss of ventilation shaft efficiency. Given the fact that most of the steel covering is subjected to considerable loads, several designs have been developed that take the safety of steel coverings into consideration.

However, a systematic optimal design methodology for steel covering has not yet been developed, and in most cases, these coverings have been designed simply based on experience. This has led to several problems that are due to the large deflection or rupturing of the ribs that support the steel covering under unexpected overloads. As reported in The Law \& Police News [2], 27 people who had climbed atop an aboveground ventilation facility to witness a recent live concert in Seoul, Korea, either lost their lives or were seriously injured when the steel covering collapsed, which was caused by accidental overloading on the ventilation shafts. An investigation of this accident was conducted, and the results indicated that the collapse was attributed to overloading on the stiffening ribs of the ventilation shaft, not because of any defect in the top plate. If we consider the weight of one person to be $70 \mathrm{~kg}$ on average, the total weight of 27 people would be $1,890 \mathrm{~kg}$. Although such a load is not 


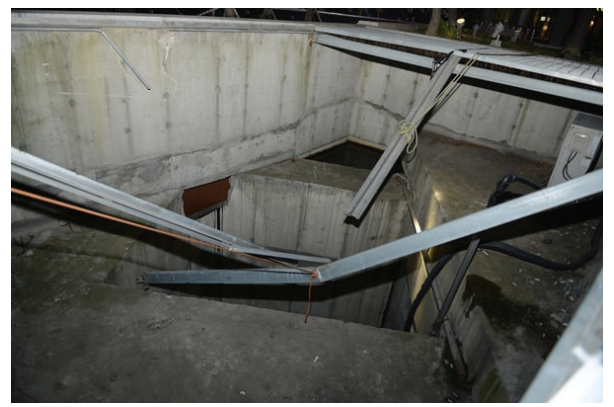

FIGURE 1: An accident that occurred in 2014 involving the collapse of ventilation covering [2].

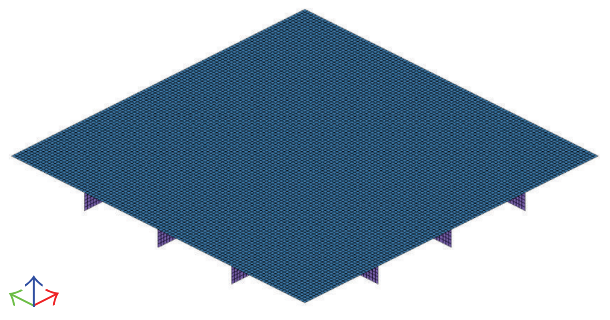

(a) Top view

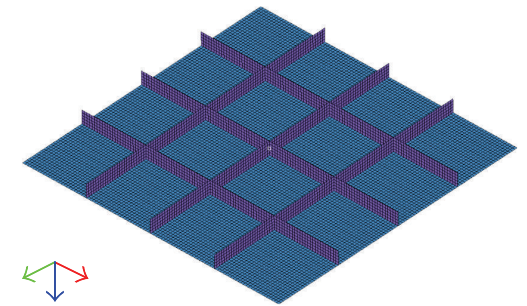

(b) Bottom view

FIGURE 2: Top and bottom isometric views of the steel ventilation covering.

considered problematic given that the ventilation covering is made of steel, which can withstand greater loads, the investigation revealed that the ventilation covering collapsed because the stiffening ribs were insufficiently designed. A picture of the collapse is shown in Figure 1.

To design this kind of structure safely and optimally, the positions and dimensions of ribs are most critical. A procedure of finite element analysis (FEA) was developed for varying locations and thickness of the ribs in order to acquire their minimum total weight while satisfying the stress constraint (and the strength constraint against maximum deflection, if necessary). Furthermore, in research by Coello and Pulido [3], the micro genetic algorithm (MGA) was used as an effective multipurpose optimization method to obtain a global optimal solution. The MGA is suitable for solving optimization problems globally with a relatively small number of individuals. Additionally, the special genetic algorithm (SZGA [4]) was proposed to accelerate the convergence rate particularly at the later stage of optimization.

The positional set representing the locations of ribs is not a continuous variable; hence, it is mapped to a dimensionless position variable. A certain interval of position variables is matched to a certain set of positions. Therefore, there is no change in the locations of ribs through that interval of position variables, and the objective function of overall weight of the structure is constant. Therefore, the objective function is a stepwise function along with the position variable. In order to quickly extract a more optimal solution using this genetic algorithm, the stepwise objective function was modified to be a smooth interpolated function using a FORTRAN code. The convergence rates for the original and modified objective functions will be compared.

\section{Discrete Optimization Problem}

The conventional optimization of steel ventilation coverings is easily determined by adjusting the thickness of all of the ribs. However, it is technically difficult to optimize the locations of ribs. The overall shape of the steel ventilation covering is illustrated in Figure 2, where the top cover is represented by a top plate. As shown in this figure, basic supports exist at the peripherals, and the ribs are connected in-between to support the steel cover. The location of a rib is hard to be continuously changed because of remeshing problems; therefore, we provided two spare ribs on both sides of a basic rib, where their primary locations were determined from design experience, as illustrated in Figure 3. Therefore, the objective of location optimization is to determine the optimal locations for a total of six groups, selecting one rib in each group.

However, in the case of this model, the number of possible combinations becomes considerably large when three locations exist for each group; therefore, this model will be simplified for execution. The ribs within ventilation shaft are support beams that dominantly endure the load applied to the steel covering. Therefore, a small change in the location or thickness of a rib not only brings about a considerable change in the stress occurring in the steel ventilation covering but may also causes an excessive deflection or rupture of the entire structure. The objective of this study is to acquire the optimal locations and thickness of ribs that satisfy a reliable safety margin while minimizing their total weight.

2.1. Design Constraints. The von Mises equivalent stress (and maximum displacement, if necessary) was the constraint 


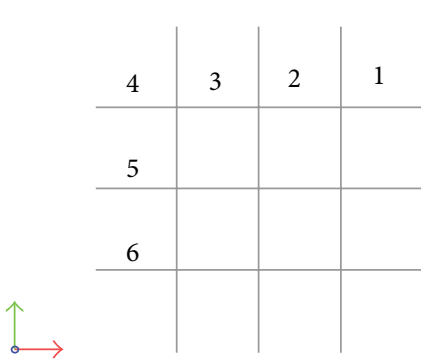

(a)

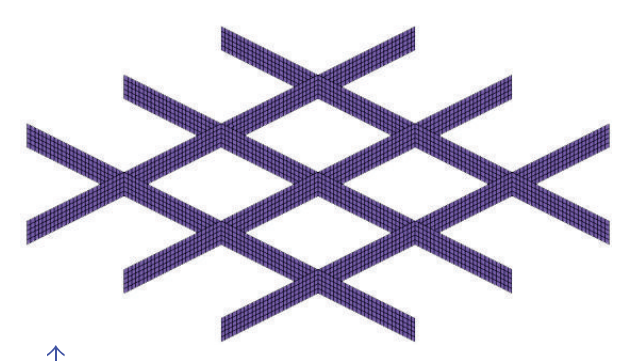

(b)

FIgURE 3: Locations and shapes of basic ribs.

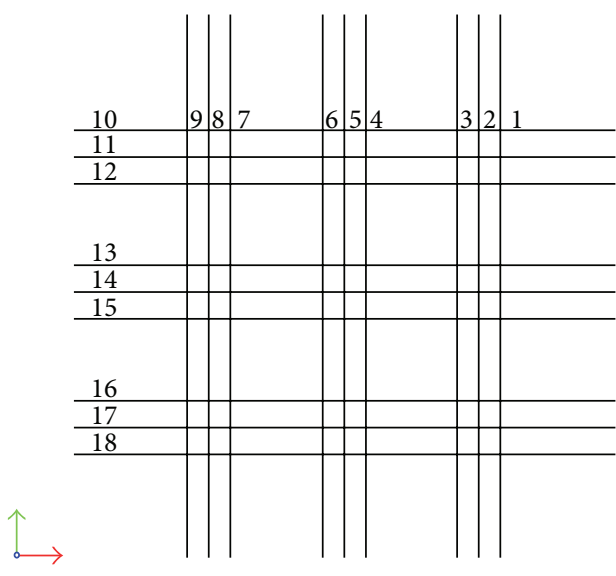

(a)

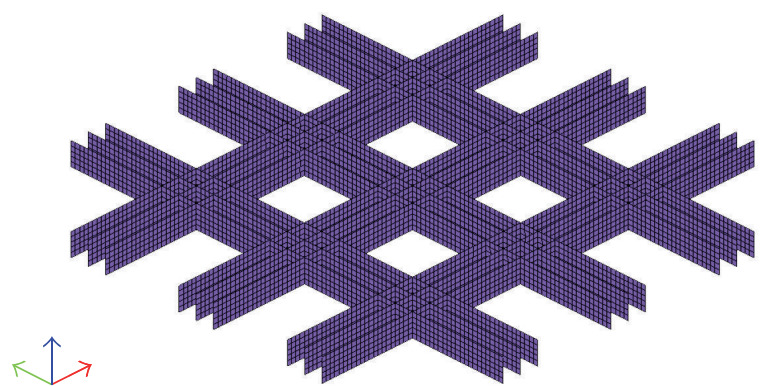

(b)

FIGURE 4: Model of three ribs in each group to find the best position.

conditions considered in this study. In particular, a stress of $50 \mathrm{MPa}$ that satisfies a safety factor of 5 is considered to be the constraint condition required in this study.

2.2. Ribs of Steel Ventilation Covering. The basic design of steel ventilation covering includes six ribs at the initial locations of $2,5,8,11,14$, and 17 , located at the center of each group, as illustrated in Figure 4. In this study, the most effective and optimal support positions for the six groups were acquired in order to determine the minimum weight of steel ventilation covering. Furthermore, in order to avoid remeshing the steel ventilation covering after changing the rib location, the use of two additional ribs (illustrated in Figure 4) was suggested parting pregiven distances from the primary position. The accuracy of the design can be improved by increasing the number of positions in each group, with smaller gaps between them. Subsequently, attempts were made to determine the optimal location of the ribs, one for each group. For further improvement, the entire process can be repeated with a narrower group whose central location is at or closer to the optimal location determined in the previous stage.
2.3. Design Variables. As mentioned previously, the objective of this study is to determine the most appropriate locations and optimal thickness for ribs of ventilation covering in order to minimize their total weight. In order to acquire an optimal position set of discrete variables, a unique rib position variable was designated for the positional set as was done by Lee et al. [5]. With regard to this continuous variable for the discrete positions of ribs, a response based on the position variable does not continuously change but changes at the discontinuities in the objective function.

A considerable amount of time would be required to analyze the $729\left(=3^{6}\right)$ cases required to optimize the three possible locations of ribs in six groups. Therefore, the analysis and optimization were carried out using a simplified model (2nd quadrant of Figure 4), as illustrated in Figure 5, taking advantage of the symmetrical structure of the steel ventilation covering. As a result, the number of cases was reduced to nine $\left(=3^{2}\right)$. Therefore, nine kinds were assigned to the possible locations in advance. Furthermore, one position was selected from each group as a set of the two groups (i.e., one in 79 , one in 10-12, with 5 and 14 fixed). The dimensionless rib position variable $z$ is set within the range of $[0,1]$, and 9 position combinations were mapped to the range of $[0,1]$. 


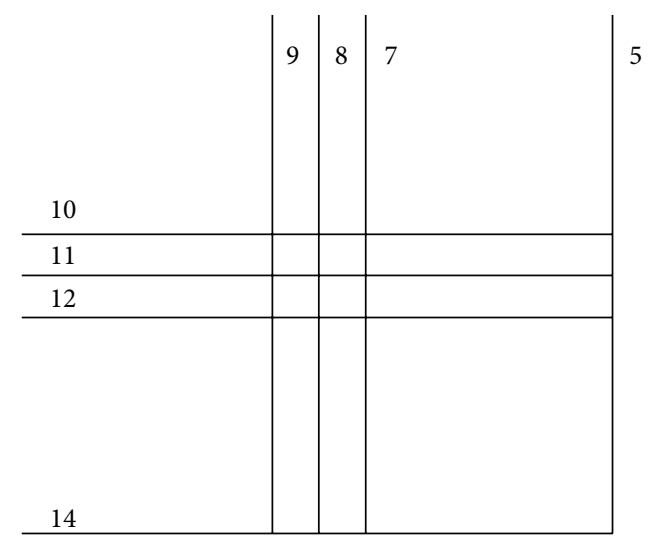

FIGURE 5: Simplified model considering the symmetry of the structure.

Each interval represents a particular position set number. For example, $0 \leq z \leq 1 / 9$ corresponds to the first set $(7,10)$.

2.4. Interpolation Model for Optimal Design. In this study, the rib position variable denotes a list of unrelated and discontinuous position sets. The rib position number associated with each position set is a value given by the user. For this example, if the quantity $z$ is in the range $[0,1 / 9]$, the corresponding set of positions is $(7,10)$, or $(5,7,10,14)$ when including the fixed ribs. In a range allocated to a given set, the positions of the ribs are the same, which results in the same objective function of the weight. The MGA is a global search algorithm that works very effectively for this kind of step or piecewise continuous function. In addition, we also adopted the interpolated continuous objective function shown in Figure 6 for the MGA, which gives better convergence than the original step objective function. It may also be used in a gradient search algorithm or in a hybrid algorithm of the MGA followed by a local gradient search for an exact optimum with better convergence.

The MGA is used in this manner for the optimization of the locations as well as the thickness of the ribs for both types of objective functions. According to PIDOtech [6], the MGA uses a smaller number of samples than the genetic algorithm. Therefore, although the MGA exhibits relatively prompt convergence, the possibility of early convergence to a near optimum still exists because commercial optimization packages prioritize convergence, and the MGA is only appropriate for problems with a small number of variables without any constraints. To interface with the MGA, an approximate function was developed in FORTRAN. When only the MGA is used, there exists a possibility that it may not enter the domains of discrete locations with better solutions; however, a more accurate search result can be determined when optimizing with an interpolated continuous objective function.

Therefore, an interpolated continuous model is developed, as in Figure 6, with the weight being based on the rib position variable created through sampling. This function can be used with the MGA to effectively find better genes, and it enables accurate calculations while satisfying the required

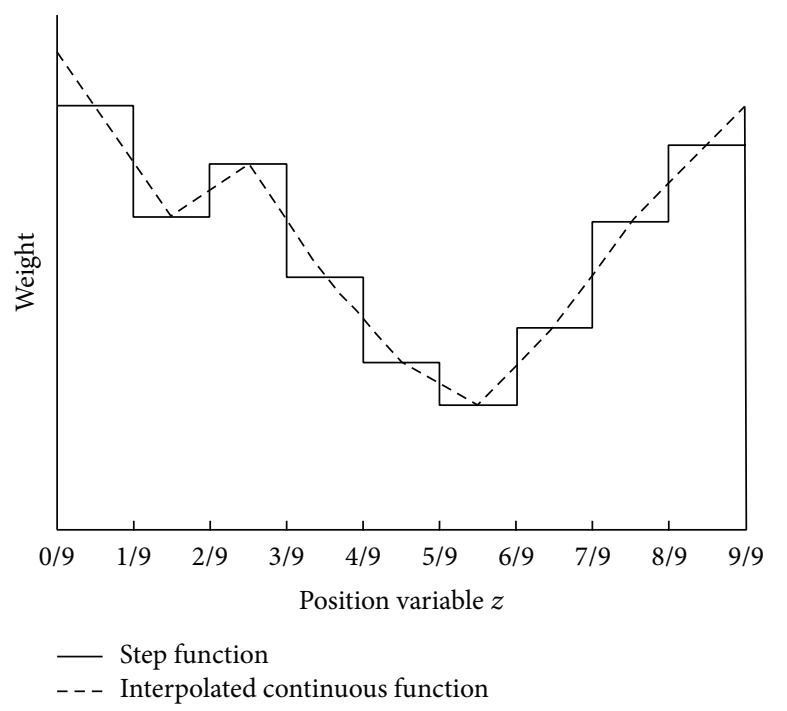

FIGURE 6: Schematics of step and interpolated continuous objective functions used with the MGA.

constraints of the system. In this study, the positional optimization is performed using the MGA alone and with a continuous function for comparison.

2.5. Formula for Optimal Design. There are five design variables: the thickness $x_{i}$ and the dimensionless number $z$. The five design variables $\left(z, x_{5}, x_{\beta}, x_{\gamma}\right.$, and $\left.x_{14}\right)$ can be expressed as the solution vector $X=\left(z, x_{\alpha}, x_{\beta}, x_{\gamma}, x_{\delta}\right)^{T}$. The positions $\beta$ and $\gamma$ depend upon the variable $z$. The formula for the optimal design of the rib of the steel ventilating covering is designed to minimize the overall weight of the ribs while satisfying the design constraints. The optimization problem is expressed in

$$
\text { find } \mathbf{X}=\left(z, x_{5}, x_{\beta(z)}, x_{\gamma(z)}, x_{14}\right)^{T} \text {, }
$$

to minimize $W_{\text {rib }}$,

$$
\begin{array}{ll}
\text { subject to } & \sigma_{\max } \leq \sigma_{\text {allow }} \\
& x_{i}{ }^{L} \leq x_{i} \leq x_{i}^{U}, \quad i=5, \beta, \gamma, 14, \\
& 0 \leq z \leq 1 .
\end{array}
$$

The dimensionless rib position $z$ can take values between zero and one and refers to the discretization position set; $x_{5}, x_{\beta}, x_{\gamma}, x_{14}$ refer to the thicknesses of the ribs at positions $(5, \beta, \gamma, 14)$, where the 5 th and 14 th positions are fixed because of the symmetry. The first position number $\alpha$ of the position set is fixed as 5 , the second position number $\beta$ is selected from group 7-9, the third position number $\gamma$ is selected from group 10-12, and the last position number $\delta$ is fixed as 14 (see Figure 5). The maximum von Mises stress of the structure is represented with $\sigma_{\max }$. The allowable stress $\sigma_{\text {allow }}$ is the yield strength divided by a safety factor of 5 . All thickness values are constrained by their maximum or minimum values. The symbols $x_{i}{ }^{L}$ and $x_{i}^{U}$ refer to the minimum and maximum 


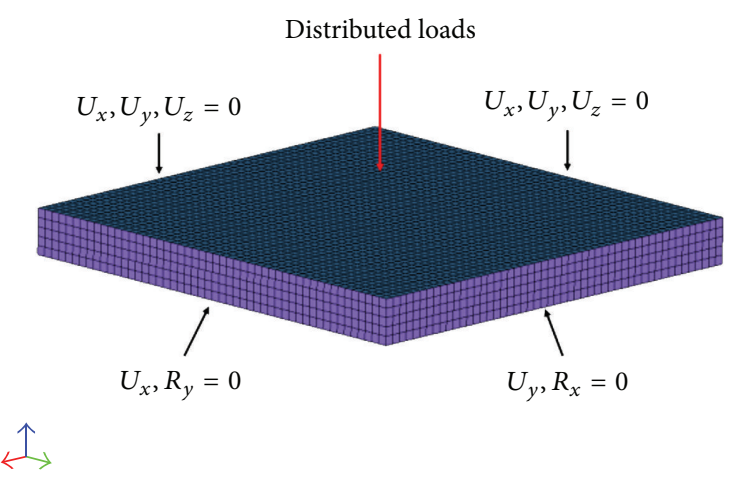

FIGURE 7: Boundary conditions and meshing of the steel ventilation covering (front 14 th and 5 th ribs).

rib thickness, which are determined from the inventory specification or manufacturing efficiency.

\section{Automatic Optimization Processor Using PIAnO}

PIAnO is a commercial package for executing process-integrated optimal design. It changes design variables for an objective function, executes finite element analyses, conducts a test of the objective function, checks the constraint conditions, and repeats this process as necessary to find an optimal solution. The use of this package was previously demonstrated by Lee et al. [7].

3.1. Finite Element Analysis. First, a 3D model of the steel ventilation covering and support was created using Pro-Engineer. Before the FEA began, mesh creation, boundary-condition allocation, and analysis stage setting were completed using the commercial package Hypermesh as illustrated in Figure 7. Static structural analysis was conducted using the commercial package Abaqus.

A rib is placed at a certain location in each group during the optimization process (see Figure 5). Although there are six sets of elements, each with different attributes for each rib, they may not be used simultaneously; ribs of unselected locations can be disabled through the on/off command of the component using the Hypermesh cmf scripter prepared for the finite element model of the ribs. In other words, the thickness attributes of the unselected sets of elements are assigned to be zero.

3.2. Optimization Process. The entire process begins by creating a model of the steel ventilation covering using a computer aided design tool. Then, the preconditioning process is executed using Hypermesh. The cmf file of Hypermesh is used to designate the location and thickness of a rib based on the stepwise or interpolated continuous objective function and is used as input file arranged by PIAnO as illustrated in Figure 8. The Abaqus input file is sent from Hypermesh and used by the Abaqus solver. A FORTRAN code artificially maps the position number $z$ to the position set to change the location by changing the attributes of the elements. Therefore,

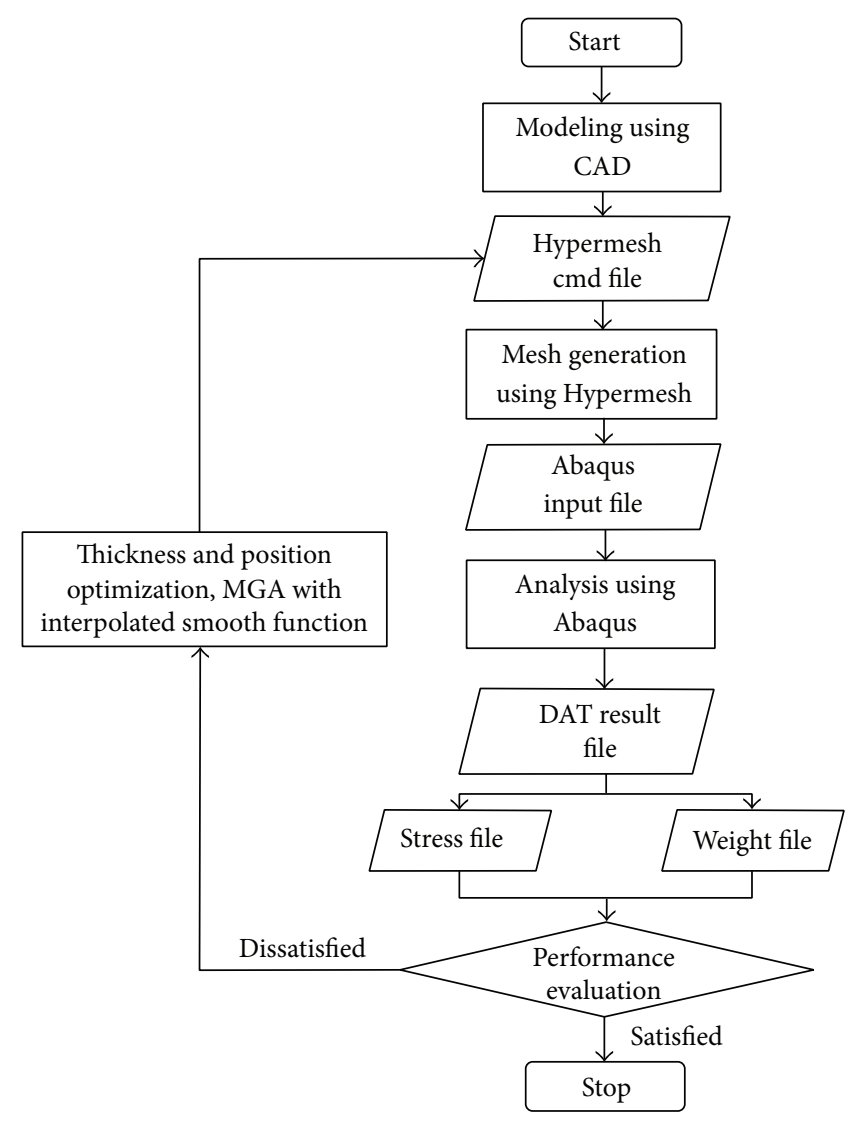

FIGURE 8: Integrated optimal design procedure.

three batch files are created to implement the processes of Abaqus, Hypermesh, and FORTRAN. The optimal thickness and locations of the ribs are acquired based on the strengths of ribs, the safety margin, and the constraint to minimize the weight by referring to (1). Although it is a stepwise discrete problem owing to the discrete position of the ribs, the MGA method with an interpolated continuous objective function is used in this study to improve convergence while preserving the capability of a global search.

\section{Optimization Results}

After completing the optimization process, an optimal solution satisfying all of the imposed requirements was acquired. As summarized in Table 1, the safety factor of the structure is 5 with regard to the yield stress of SM490. As shown in Figure 9, the maximum von Mises stress evaluated for the structure using MGA with an interpolated continuous objective function is $49.930 \mathrm{MPa}$, which is very close to the upper limit of $50 \mathrm{MPa}$. As a result, it was revealed that the optimal position set of the ribs is $(5,7,12,14)$, with thicknesses of $7.062 \mathrm{~mm}, 4.859 \mathrm{~mm}, 3.496 \mathrm{~mm}$, and $7.778 \mathrm{~mm}$, respectively. Considering the entire model from the results in Table 1, there was a $42.05 \%$ decrease in the rib weight from the weight of $0.195 \mathrm{Mg}$ under the initial conditions to $0.113 \mathrm{Mg}$.

The source of this accelerated convergence is as follows. In common genetic algorithms (GA) applied to continuous 
TABLE 1: Optimization results of each method.

\begin{tabular}{lccccc}
\hline Evaluation index & Design parameters & Initial state & $\begin{array}{c}\text { Fixed position } \\
\text { (gradient) }\end{array}$ & $\begin{array}{c}\text { MGA } \\
\text { (step function) }\end{array}$ & $\begin{array}{c}\text { MGA } \\
\text { (interpolated continuous function) }\end{array}$ \\
\hline \multirow{2}{*}{ Design variables } & Thickness $\left(x_{\alpha}, \mathrm{mm}\right)$ & 10 & 9.244 & 9.043 & 7.062 \\
& Thickness $\left(x_{\beta}, \mathrm{mm}\right)$ & 10 & 3.249 & 4.313 & 4.859 \\
& Thickness $\left(x_{\gamma}, \mathrm{mm}\right)$ & 10 & 3.249 & 4.983 & 3.496 \\
& Thickness $\left(x_{\delta}, \mathrm{mm}\right)$ & 10 & 9.244 & 6.265 & 7.778 \\
& Position set $(\alpha, \beta, \gamma, \delta)$ & $(5,8,11,14)$ & $(5,8,11,14)$ & $(5,8,12,14)$ & $(5,7,12,14)$ \\
\hline Objective & Weight $\left(W_{\text {rib }}, \mathrm{Mg}\right)$ & 0.195 & 0.122 & 0.119 & 0.113 \\
\hline \multirow{2}{*}{ Constraints } & von Mises stress $(\sigma, \mathrm{MPa})$ & \multirow{2}{*}{33.430} & 45.400 & 49.100 & 49.930 \\
\hline
\end{tabular}

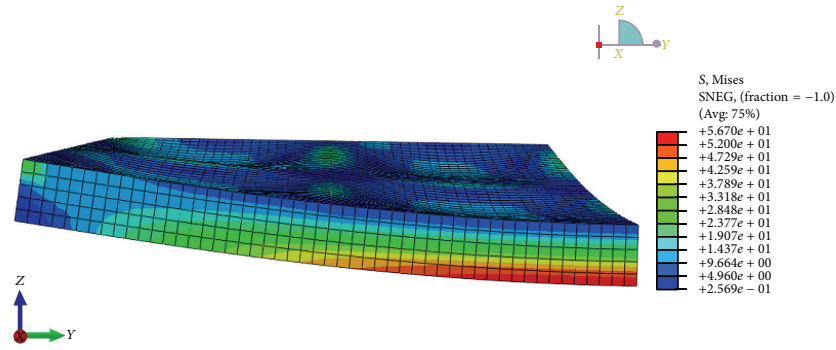

(a) Side view

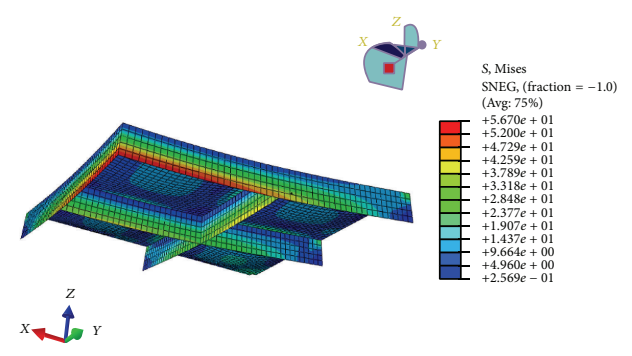

(b) Bottom view

FIGURE 9: von Mises stress distribution in the steel ventilation covering.

objective functions, the chromosomes of parents of good fitness are mixed to produce the offspring. Some offspring are located farther from the parents, but more are located near the parents. After many generations, offspring are more closely located near the optimal solution. For this case of a continuous objective function, the ratio of offspring with poor fitness to those with good fitness is relatively moderate. The ratio would decrease with progressed generations.

When one uses the stepwise objective function, a selected parent located near the boundary of the constant function would produce many offspring of poor fitness along with many offspring of good fitness. The ratio of offspring with poor fitness to good fitness may be relatively large. The ratio would not decrease with progressed generations, because the parents are near the fitness cliff as long as they are located near the boundary of the constant objective function.

In short, the use of the proposed interpolated smooth model removes the chance of individuals, located near the boundary of the constant objective function, being selected as parents for the next generation. With a constant objective function, individuals near the boundary have the same fitness as individuals located near the center of the division; however, with the interpolated smooth model, individuals near the boundary have lower fitness compared to individuals near the center of the division. Therefore, most parents are far from the fitness cliff, which accelerates convergence to the optimal solution. Finally, the interpolated smooth model is continuous and can be used in the gradient method of optimization from the first stage-or from a later stage where the convergence rate is very slow in most GA-to pinpoint the optimal solution in a hybrid manner.

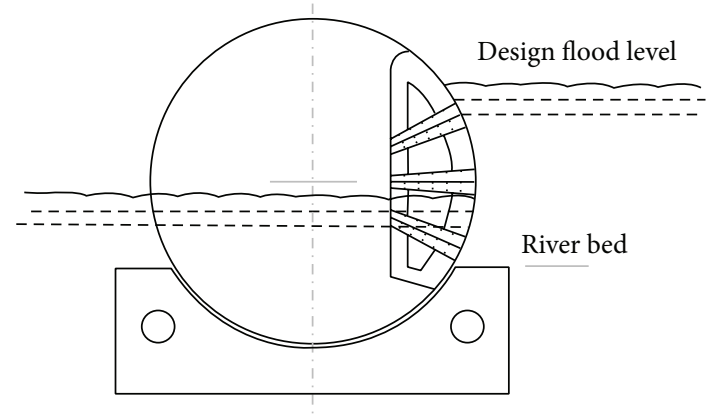

Flood control position

FIGURE 10: Schematics of the rising sector gate.

\section{Verification Using an Alternative Example Structure}

Using a continuous function with MGA improves the accuracy for the position and thickness optimization problem of the steel ventilation covering. For verification, we also applied this method to another problem, which was studied by Lee et al. [5]. The problem is an optimization of a rising sector gate as illustrated in Figure 10. The objective of this optimization example is to find the position of three girders among nine possible locations and the thickness of each girder. The objective function is the minimization of the total weight. The constraints are a maximum von Mises stress of 119.68 MPa, a maximum deflection of $53 \mathrm{~mm}$, and a minimum 
TABLE 2: The optimal solution using MGA with an interpolated continuous objective function.

\begin{tabular}{|c|c|c|c|}
\hline Evaluation index & Design parameters & Initial state & $\begin{array}{c}\text { MGA } \\
\text { (interpolated continuous function) }\end{array}$ \\
\hline \multirow{4}{*}{ Design variables } & Thickness $\left(x_{\alpha}, \mathrm{mm}\right)$ & 20 & 12.478 \\
\hline & Thickness $\left(x_{\beta}, \mathrm{mm}\right)$ & 20 & 25.825 \\
\hline & Thickness $\left(x_{\gamma}, \mathrm{mm}\right)$ & 20 & 6.445 \\
\hline & Girder position set & $(2,5,8)$ & $(3,4,9)$ \\
\hline Objective & Weight $\left(W_{\text {girder }}, \mathrm{Mg}\right)$ & 94.293 & 69.192 \\
\hline \multirow{3}{*}{ Constraints } & $\begin{array}{l}\text { von Mises stress } \\
(<119.67 \mathrm{MPa})\end{array}$ & 118.993 & 119.46 \\
\hline & $\begin{array}{l}\text { Lateral displacement } \\
\qquad(<53.000 \mathrm{~mm})\end{array}$ & 52.701 & 52.453 \\
\hline & $\begin{array}{l}\text { 1st frequency } \\
(>10.5000 \mathrm{~Hz})\end{array}$ & 10.526 & 10.526 \\
\hline
\end{tabular}
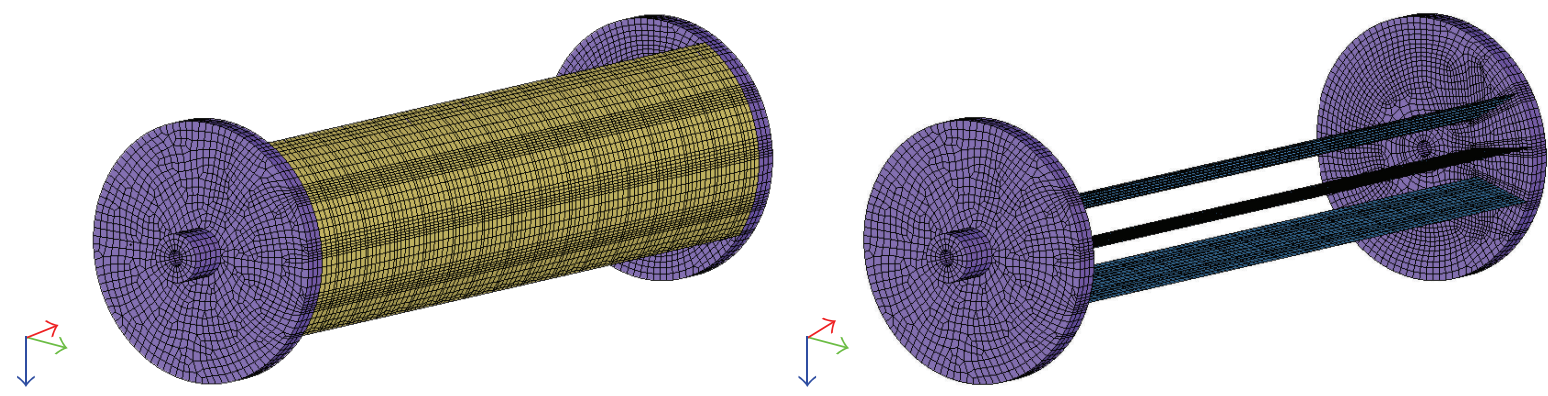

FIGURE 11: Meshes of the finite element model.
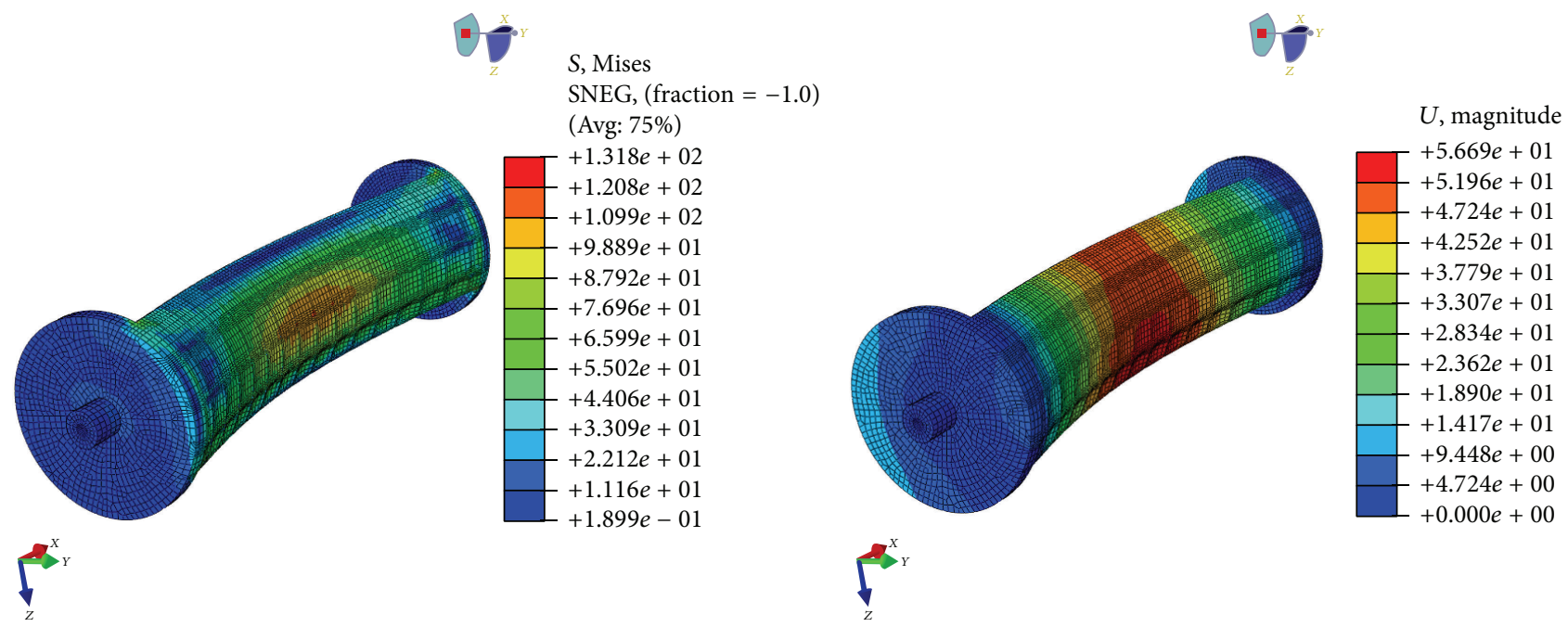

FIGURE 12: Finite element result of the rising sector gate.

natural frequency of $10.5 \mathrm{~Hz}$. The shape of the finite element model is shown in Figure 11.

The results of the optimization are shown in Table 2. The von Mises stress and displacement distribution are shown in Figure 12. As summarized in Table 2, the result of the initial model is compared with the result of the optimal result produced by the MGA with an interpolated continuous objective function. It satisfies the three constraint conditions and reduces the weight by $26.6 \%$ compared to the initial empirical model. In this example, the MGA with 
an interpolated smooth objective function very effectively obtained an optimal solution despite the size and complex constraint conditions of this actual design problem.

\section{Conclusion}

The issues of discrete optimal design were evaluated in this study in order to minimize the weight of ribs for steel structures while satisfying various constraints. The optimization process was integrated using PIAnO, Hypermesh, Abaqus, and a FORTRAN code. The discrete optimization problem can be converted into a continuous variable problem, which resulted in a stepwise objective function problem. This stepwise objective function was modified to give an interpolated continuous function, which provides the ability for the MGA to possess fast global search characteristics.

The proposed conversion, modifying techniques, and the analysis results can be summarized as follows.

(1) The conversion technique of mapping a discrete position set into a continuous position variable was applied to design structures optimally by using the MGA with an interpolated continuous objective function.

(2) The MGA with an interpolated continuous objective function was used to effectively improve the convergence to the optimal structure design with the given constraints. It may be extended to a local gradient search-or a hybrid algorithm of the MGA followed by a local gradient search algorithm.

(3) An analysis of the ribs of ventilation covering was conducted to acquire the optimal solution, and it was revealed that the thicknesses of each rib in the optimal position set $(5,7,12,14)$ were $7.062 \mathrm{~mm}, 4.859 \mathrm{~mm}$, $3.496 \mathrm{~mm}$, and $7.778 \mathrm{~mm}$, respectively. The MGA with an interpolated continuous objective function obtained a more accurate solution than the MGA with a stepwise objective function.

(4) The optimization method applied to the ventilation covering yielded a $42 \%$ decrease in the total weight of the ribs while satisfying all imposed constraint conditions in comparison with the initial guess. In the previously constructed rising sector gate case, the optimization method decreased the weight by $27 \%$ with respect to the initial state.

\section{Conflict of Interests}

The authors declare that there is no conflict of interests regarding the publication of this paper.

\section{Acknowledgments}

This research was supported by Kyungpook National University Research Fund, 2012. The authors are partially supported by the Brain Korea 21 Plus project of the Korean Research Foundation.

\section{References}

[1] Y. Lee, T.-O. Lee, Y.-J. Um, and C. Choi, “The basic design notion for steel grate of road drainage," in Proceedings of the Korean Geo-Environmental Conference, 2007.

[2] The Law \& Police News, 2014, http://www.112news.co.kr/news/ content.asp?news_idx $=201410271540438085$.

[3] C. A. C. C. Coello and G. T. Pulido, "A micro-genetic algorithm for multi-objective optimization," in Evolutionary Multi-Criterion Optimization, vol. 1993 of Lecture Notes in Computer Science, pp. 126-140, Springer, 2001.

[4] Y.-D. Kwon, S.-B. Kwon, S.-B. Jin, and J.-Y. Kim, "Convergence enhanced genetic algorithm with successive zooming method for solving continuous optimization problems," Computers \& Structures, vol. 81, no. 17, pp. 1715-1725, 2003.

[5] K. Lee, H. Gil, X. Lu, and Y. Kwon, "A discrete optimization process for the design of horizontal girders of rising sector gate using PIAnO," International Journal of Steel Structures, vol. 14, no. 3, pp. 601-607, 2014.

[6] PIDOtech, PIAnO (Process Integration, Automation and Optimization) User Manual, Version 3.3, 2011.

[7] G. Lee, J. Park, and D.-H. Choi, "Shape optimization of mobile phone folder module for structural strength," Journal of Mechanical Science and Technology, vol. 26, no. 2, pp. 509-515, 2012. 


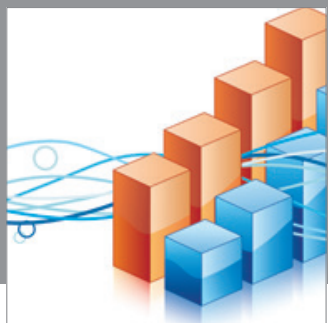

Advances in

Operations Research

mansans

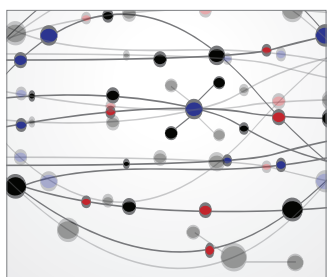

The Scientific World Journal
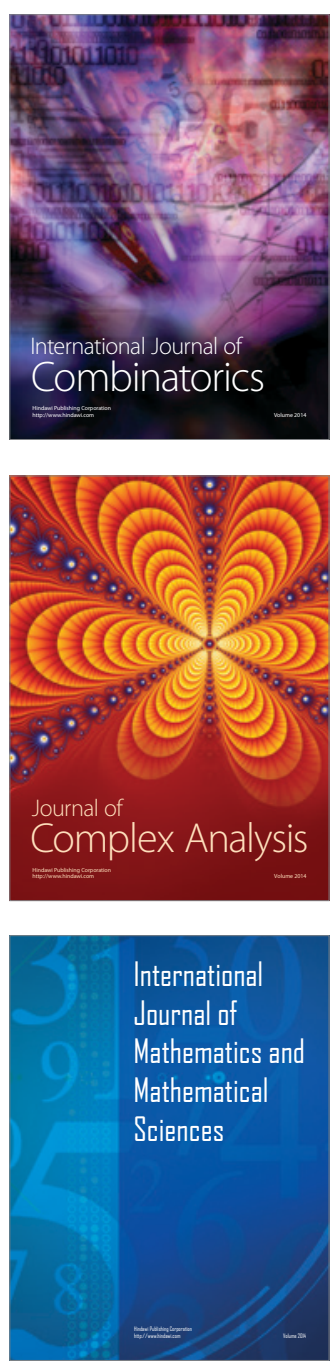
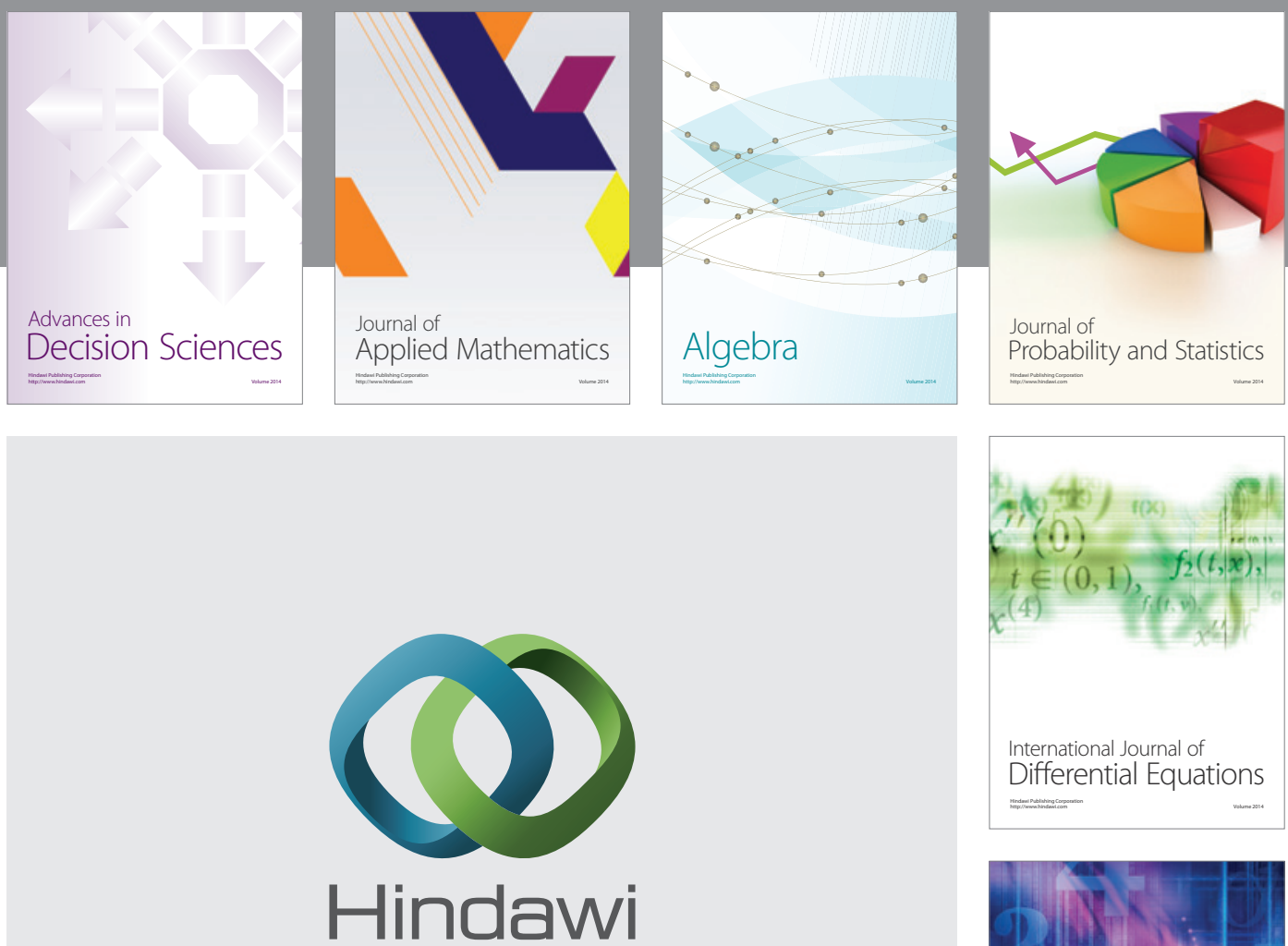

Submit your manuscripts at http://www.hindawi.com
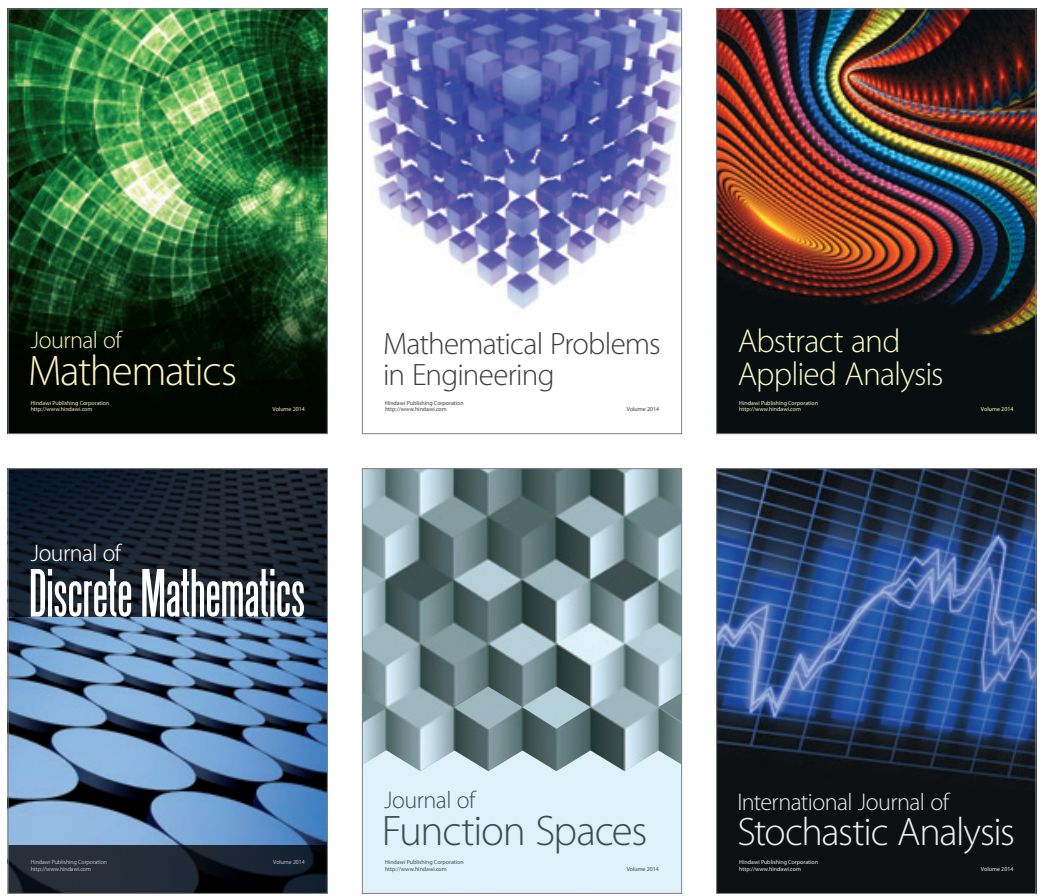

Journal of

Function Spaces

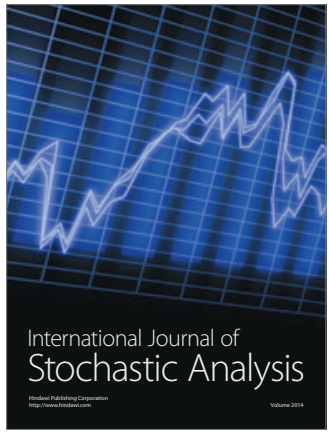

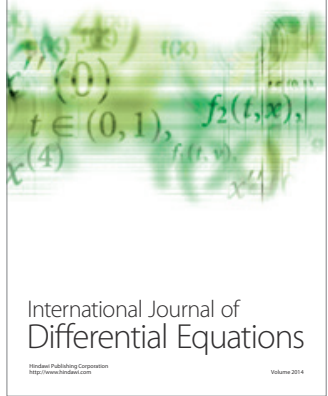
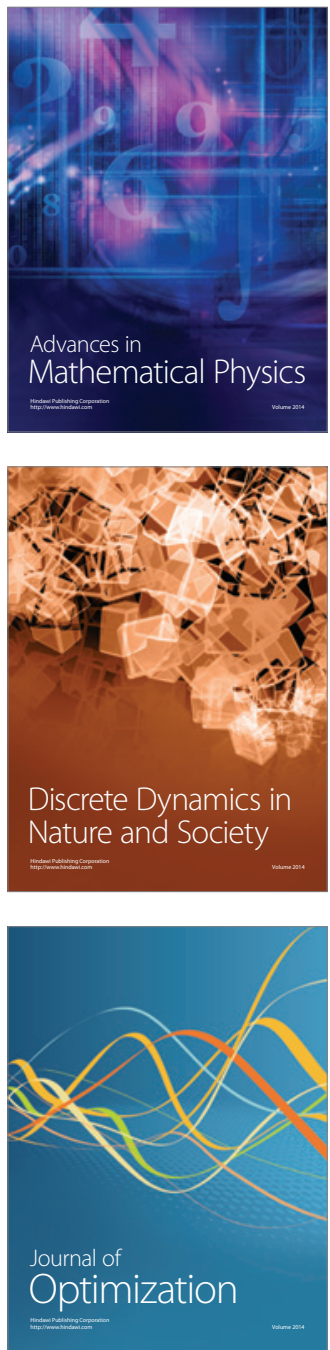